\title{
A Rare Case of ocular cystinosis with systemic involvement
}

\author{
Thakkar $\mathrm{UH}^{1 *}$, Solanki $\mathrm{AH}^{2}$, Ganvit $\mathrm{SS}^{3}$ \\ 1.PG resident, Department of Ophthalmology, SSG hospital,Vadodara \\ 2.Tutor, Department of Ophthalmology, SSG hospital,Vadodara \\ 3. Head Of Department, Department of Ophthalmology, SSG hospital,Vadodara
}

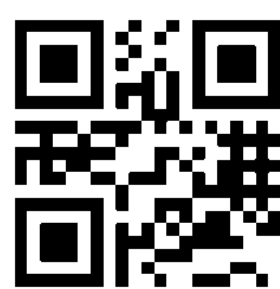

\section{Abstract :}

A 13 year old female came to ophthalmic opd on 17/01/2019 at S.S.G. Hospital, Vadodara with complaints of dimness of vision and severe photophobia in both eyes. Patient is a known case of Nephrogenic Cystinosis with Hypothyroidism with Rickets with ocular cystinosis since 2013 and on systemic medication and cystinosine eds. Slit lamp examination shows crystalline deposition in corneal stroma and subepithelium of palpebral conjunctiva. D fundus examination shows B/E Pale Disc ( R/E > L/E ) with RPE mottling present with multiple yellowish flecks cystine crystal present in RPE layer. Genetic test shows positive result for homozygous pathogenic variant identified in CTNS C 18-21 del GACT in CTNS

.Key words: Ocular Cystinosis, Cysteamine Eye Drops, RPE Mottling, CTNS Gene

\author{
*Corresponding Author \\ Dr. Umang H. Thakkar
}

E-mail id: umangthakkar131192@gmail.com

Copyright: This is an open-access article distributed under the terms of the Creative Commons Attribution license which permits unrestricted use, distribution, and reproduction in any medium for non-commercial use (Non Commercial, or CC-BY-NC) provided the original author and source are credited.

\section{INTRODUCTION}

Cystinosis is a rare, incurable metabolic autosomal recessive genetic disease that affects male and female in equal number. The disorder is estimated to occur in 1 in $1,00,000-2,00,000$ people in the general population approx. only 2000 cases diagnosed worldwide. ${ }^{1-2}$ Cystinosis is lysosomal storage disease caused by mutation in CTNS gene on chromosome 17p13 which results in accumulation of amino acid cystine in kidneys, eyes, muscles, pancreas, brain, etc.

\section{CASE REPORT}

A 13 year old Indian female came to ophthalmic OPD in S.S.G. hospital on 17/01/2019 for visual assessment in $\mathrm{k} / \mathrm{c} / \mathrm{o}$ Nephrogenic Cystinosis for Disability Certificate. At age of 7 years, patient was diagnosed Nephrogenic Cystinosis with chronic kidney disease in AIIMS Hospital Delhi and ocular examination was done there. Visual acuity in both eyes was noted $6 / 18$ and with best corrected visual acuity in both eyes was 6/12.On slit lamp examination, both eyes shows crystal deposition in stroma at cornea and subepithelium of palpebral conjunctiva. $\mathrm{B} / \mathrm{E}$ dilated fundus examination shows pale disc, yellowish flecks, retinal pigment epithelium mottling. IOP measured with Tonopen was within normal limit. VEP in both eyes shows a normal latency and amplitude in both eyes. ERG shows a normal response in left eye, but a delay in latency and reduced amplitude in right eye. A sample sent for genetic test shows positive result. Homozygous pathogenic variant identified in CTNS gene (positive for C18-21 del GACT in CTNS gene).General examination and investigation shows systemic involvement like Nephrogenic Cystinosis, rickets, vit. A and Fe deficiency and hypothyroidism at that time, vit. D, Fe, Po4supplementation given and cystagon eye drops started and serial LFT and RFT and ophthalmic examination advised. On subsequent follow up symptoms of photophobia increased and visual acuity decreased, progressive $\mathrm{B} / \mathrm{E}$ corneal cystine deposition on D fundus and RPE mottling seen.

\section{RESULTS}

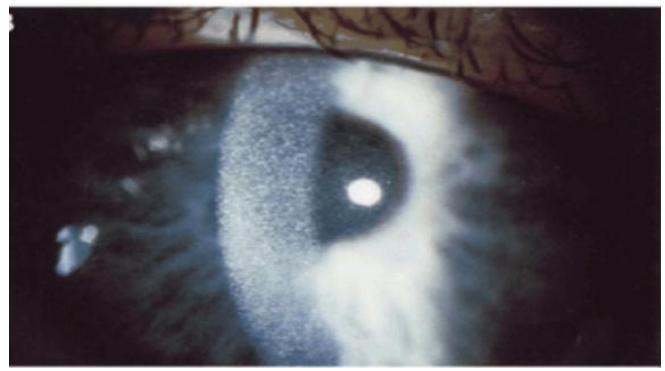

Figure1.Slit lamp examination shows crystal deposition in cornea 


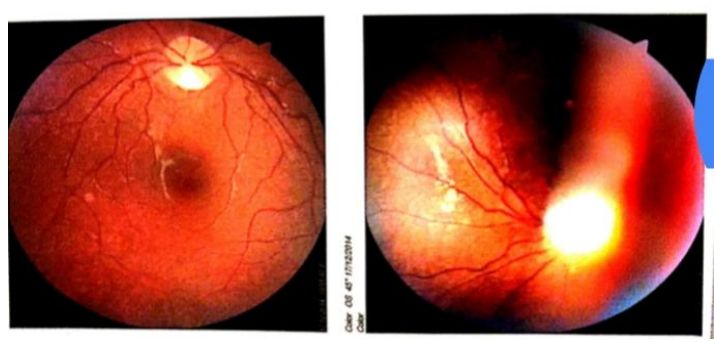

Figure 2: Fundus photographs shows both eye pale disc with crystal deposition in retina

)

Cystinosis Test

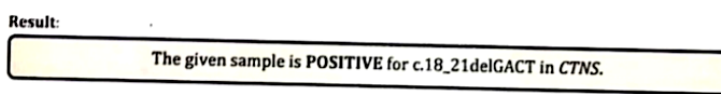

Results Summary:

- Variant iden

- Zygosity: Homozygo

- Variant classification: Pathogenic

Figure 3: Genetic test shows homozygosity positive cystinosis test for C.18-21del GACT in CTNS gene

\section{DISCUSSION}

A 13 yrs old Indian female child with diagnosed case of Nephrogenic Cystinosis has a short stature, light complextion, B/L genu vulgum with skeletal dysplasia and photophobia our patient had been diagnosed Nephrocystinosis with rickets with hypothyroidism with growth failure. Slit lamp examination showing crystal deposition in stroma of cornea and subepithelium of palpebral conjunctiva and dilated fundus examination shows RPE mottling. Genetic test shows positive result for cystinosis test sample positive for C18-21 del GACT in CTNS gene (homozygous). Anterior segment OCT and TEM can be useful for diagnosis. Patient instilled topical cysteamine eyedrops since 6 years and it has proven safe and efficient at dissolving corneal crystal and relieving the symptoms of photophobia, blepharospasm and ocular pain. Although our patient had used topical cysteamine she presented with severe photophobia and decreased visual acuity and in that case penetrating keratoplasty can be done.

\section{CONCLUSION}

Cystinosis comprises of three allelic phenotypes:1) Nephrogenic cystinosis characterized by accumulation of cystine in almost all cells, leading to cellular dysfunction with tissue and organ impairment. Signs of renal tubular Fanconi syndrome appears as early as at the age of six months, corneal crystals can be present before age of one year and are always present after age of 16 months. Prior to the use of renal transplantation and cystine- depleting therapy, the life span in nephropathic cystinosis was no longer than ten years. With these interventions, affected individuals can survive at least into the mid-forties with satisfactory quality of life.2) Intermediate cystinosis is characterized by all the typical manifestations of nephropathic cystinosis, but onset is at a later age. Renal glomerular failure occurs in all untreated affected individuals, usually between ages 15 and 25. 3) Non-nephropathic (ocular) form of cystinosis is characterized clinically only by photophobia resulting from corneal cystine crystal accumulation.

Conflicts of interest: Declared, authors have no conflict of interest

\section{Funding/Financial support: None}

\section{REFERENCES}

1. Iwata F, Kuehl EM, Reed GF, McCain LM, Gahl WA, Kaiser-Kupfer MI. A randomized clinical trial of topical cysteamine disulfide (cystamine) versus free thiol (cysteamine) in the treatment of corneal cystine crystals in cystinosis. Mol Genet Metab 1998;64:23742.

2. Gahl WA, Thoene JG, Schneider JA. Cystinosis. N Engl J Med 2002;347:11121.

3. Town M, Jean G, Cherqui S, Attard M, Forestier L, Whitmore SA, et al. A novel gene encoding an integral membrane protein is mutated in nephropathic cystinosis. Nat Genet 1998; 18: 319-24.

4. Wong VG, Lietman PS, Seegmiller JE. Alterations of pigment epithelium in cystinosis. Arch Ophthalmol 1967;77:361-9.

5. Melles RB, Schneider JA, Rao NA, Katz B. Spatial and temporal sequence of corneal crystal deposition in nephropathic cystinosis. Am J Ophthalmol 1987;104:598-604.

6. Nesterova G, Gahl WA. Cystinosis: The evolution of a treatable disease. Pediatr Nephrol 2013;28:51-9.

7. Garibaldi DC, Gottsch J, de la Cruz Z, Haas M, Green WR. Immunotactoid keratopathy: A clinicopathologic case report and a review of reports of corneal involvement in systemic paraproteinemias. Surv Ophthalmol 2005;50:61-80.

8. Kleta R, Blair SC, Bernardini I, KaiserKupfer MI, Gahl WA. Keratopathy of multiple myeloma masquerading as corneal crystals of ocular cystinosis. Mayo Clin Proc 2004;79:410-2. 